\title{
La planificación en la producción y su incidencia en la optimización de los procesos
}

\section{Production planning and its impact on process optimization}

1 Luis Enrique Soto Chávez Universidad de Guayaquil luis.sotoc@ug.edu.ec

2 José William Ugalde Vicuña Universidad de Guayaquil jose.ugaldevi@ug.edu.ec
D https://orcid.org/0000-0001-8854-6881

https://orcid.org/0000-0003-1240-7804

Artículo de Investigación Científica y Tecnológica

Enviado: 11/12/2021

Revisado: 27/12/2021

Aceptado: 28/01/2022

Publicado:05/02/2022

DOI: https://doi.org/10.33262/ap.v4i1.1.168

Cítese:

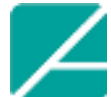

Ciencia

Digital
Soto Chávez, L. E., \& Ugalde Vicuña , J. W. (2022). La planificación en la producción y su incidencia en la optimización de los procesos. AlfaPublicaciones, 4(1.1), 411-426. https://doi.org/10.33262/ap.v4i1.1.168

ALFA PUBLICACIONES, es una Revista Multidisciplinar, Trimestral, que se publicará en soporte electrónico tiene como misión contribuir a la formación de profesionales competentes con visión humanística y crítica que sean capaces de exponer sus resultados investigativos y científicos en la misma medida que se promueva mediante su intervención cambios positivos en la sociedad. https://alfapublicaciones.com

La revista es editada por la Editorial Ciencia Digital (Editorial de prestigio registrada en la Cámara Ecuatoriana de Libro con No de Afiliación 663) www.celibro.org.ec

Esta revista está protegida bajo una licencia Creative Commons Attribution Non Commercial No Derivatives

4.0 International. Copia de la licencia: http://creativecommons.org/licenses/by-nc-nd/4.0/ 


\section{Palabras}

planificación, producción, control, procesos, optimización. claves:

\section{Resumen}

Las organizaciones actualmente batallan a diario para subsistir en el tiempo, adaptándose a las nuevas tendencias de herramientas administrativas y de la tecnología, tratando de conseguir los objetivos planeados y por ende, conseguir tanto como el crecimiento económico como organizacional, con el fin de mantener los estándares máximos de producción que conlleven al logro de esos objetivos, para ello deben entonces, optimizar cada uno de los procesos que se llevan a cabo y que de manera directa o indirecta se relacionan con el proceso de producción, el cual representa uno de los más importantes de toda organización, puesto que es a través de este que se generan los bienes o servicios que se ofrecerán a los consumidores y que por supuesto, son los que generan el ingreso. De allí a que constantemente las organizaciones se esfuerzan en buscar y aplicar distintas técnicas que le permitan asegurar el buen funcionamiento de todos los procesos, representando la planificación un proceso medular, que permite definir cada uno de los pasos que se lleven a cabo para alcanzar las metas, así como también prevenir la detección de problemas o defectos que se presenten, a fin de corregirlos a tiempo y lograr contrarrestar cualquier contratiempo.

\section{Keywords: \\ planning, production, control, processes, optimization.}

\section{Abstract}

Organizations currently struggle daily to survive over time, adapting to new trends in administrative tools and technology, trying to achieve the planned objectives and therefore, achieve both economic and organizational growth, in order to maintain the maximum production standards that lead to the achievement of these objectives, for this they must then optimize each of the processes that are carried out and that are directly or indirectly related to the production process, which represents one of the most important of any organization, since it is through this that the goods or services that will be offered to consumers are generated and that, of course, are the ones that generate income. Hence, organizations constantly strive to seek and apply different techniques that allow them to ensure the proper functioning of all processes, planning representing a core process, which allows defining each of the steps that are carried out to achieve the objectives. goals as well as 
prevent the detection of problems or defects that arise, in order to correct them in time and manage to counteract any setback.

\section{Introducción}

Hoy en día las organizaciones se valen del uso de distintas herramientas y técnicas, así como la innovación en tecnologías, para lograr alcanzar sus metas, sin embargo, una de las herramientas que representa un punto de gran importancia dentro de ellas, es la planificación, ya que, a través de esta, las organizaciones pueden diseñar los procedimientos y estrategias que conlleven a la consecución de los objetivos, la cual es utilizada en las distintas áreas de la organización.

Una de las áreas de gran valor dentro de las organizaciones, es la encargada de controlar y dirigir la producción, ya que es en esta en la cual se elaboran los productos que serán ofrecidos a los consumidores, y por ende representa la principal fuente de generación de ingresos para las mismas.

De allí, a que esta área debe contar con planes bien conformados y diseñados, a fin de asegurar que los objetivos planteados se cumplan con un mínimo margen de error, disminuyendo las pérdidas durante el proceso.

Para ello, es necesario dar el primer paso, el cual no es otros que planificar.

La planeación forma parte del proceso administrativo, (Planificar, Organizar, Dirigir y Controlar), siendo el primer proceso que se lleva a cabo al momento de la puesta en marcha de cualquier plan, estos procesos fueron establecidos por primera vez por Henry Fayol.

Según Alvarado (2018) "la planificación se refiere a imaginar que sucederá en el futuro, y de esta forma descifrar los sucesos que se originaran en el presente con el propósito de lograr los objetivos a través de la aplicación del pensamiento analítico".

Por otro lado, Solís-Ferrer \& Cortez-Fajardo (2020), señala que planificar es esencial para lograr un buen funcionamiento de los procesos, a nivel empresarial, lo cual permite obtener una perspectiva claro sobre los objetivos fijados. Así mismo sostiene que por medio de la planificación pueden fijarse estrategias que permitan lograr, desarrollar e implementar procedimientos que permitan dirigir cada uno de los procesos a fin de alcanzar las metas fijadas.

Así mismo Encalada-Tenorio et al. (2021), define la planeación como el inicio del proceso administrativo, a través del cual se establecen los objetivos, se formulan las políticas, 
planes, estrategias, procedimientos y los métodos para llevarlo a cabo y por ende alcanzar los objetivos.

De las conceptualizaciones anteriores, se desprende, que planificar es el primer paso para diseñar y establecer los objetivos que se quieren lograr en un periodo determinado, así como también, debe contemplar las tareas y quiénes serán los responsables de llevarla a cabo, a fin de optimizar la obtención de la información cuando sea requerida.

Es importante entonces, definir aquellos términos que están relacionados directamente con el tema de la investigación.

En este sentido, se menciona que la producción es definida por Muñoz (2019), como todo proceso que transforma técnica y económicamente un diseño, planificado y controlado de entradas (inputs) o factores de producción, tales como: la mano de obra, la tecnología, materia prima, información, maquinarias, instalaciones y energía, en salidas (outputs) bienes materiales y/o servicios, los cuales generar utilidad o valor para satisfacer las necesidades de los clientes.

Así mismo Pinzón (2018), señala que la producción se refiere a la realización de una serie de pasos para llevar a cabo un proceso para elaborar un producto. Se trata de transformar materia prima y poder obtener un producto con las características deseadas, a fin de satisfacer las necesidades del consumidor.

De lo anterior, se puede decir entonces, que la producción se refiere, a la creación de un determinado producto, aplicando distintos procesos y recursos, transformándolo en lo que se desea o se planifica, siempre, con miras a satisfacer las necesidades de un consumidor en particular.

Resulta importante nombrar los elementos del costo de producción, los cuales son:

Según lo señalado por Villamarin (2021) los elementos de la producción son:

1. Materia prima: Son todos aquellos productos naturales, que son transformados en otros productos.

2. Mano de obra: Está representada por todas aquellas personas que llevan a cabo los distintos procesos de los materiales, apoyándose con las maquinarias disponibles y por la destreza, conocimiento y experiencias que posean. A cambio de estas tareas, las empresas deben cancelar un salario o sueldo a cambio de sus servicios.

3. Costos indirectos de fabricación: son los costos de materiales, insumos, servicios profesionales, servicios varios y demás gastos, que sean necesarios para la producción del producto. 
4. Departamentalización: Representa cada uno de los departamentos que existen en la empresa, y los cuales generan sus propios costos indirectos de fabricación, y su desagregación permite obtener los datos de los costos de una manera más fácil.

Por otra parte, según lo citado por López (2021), indica que cada componente se comporta como un sistema mismo, ya que posee objetivos y componentes, por ello, los sistemas de producción comprenden otros subsistemas como lo son:

- Ingeniería Industrial

- Planificación y control de la producción

- Control de Calidad

- ingeniería de servicios.

Asimismo, Mantilla \& Santos (2020), señalan que un sistema de producción, son aquellos procesos que usan los recursos para luego transformar entradas en salidas esperadas.

Igualmente indica que para que un sistema de producción marche cumpliendo lo planificado, debe contar con algunos elementos, los cuales se muestran en la figura 1.

\section{Figura 1}

Elementos de un sistema de producción o de operaciones

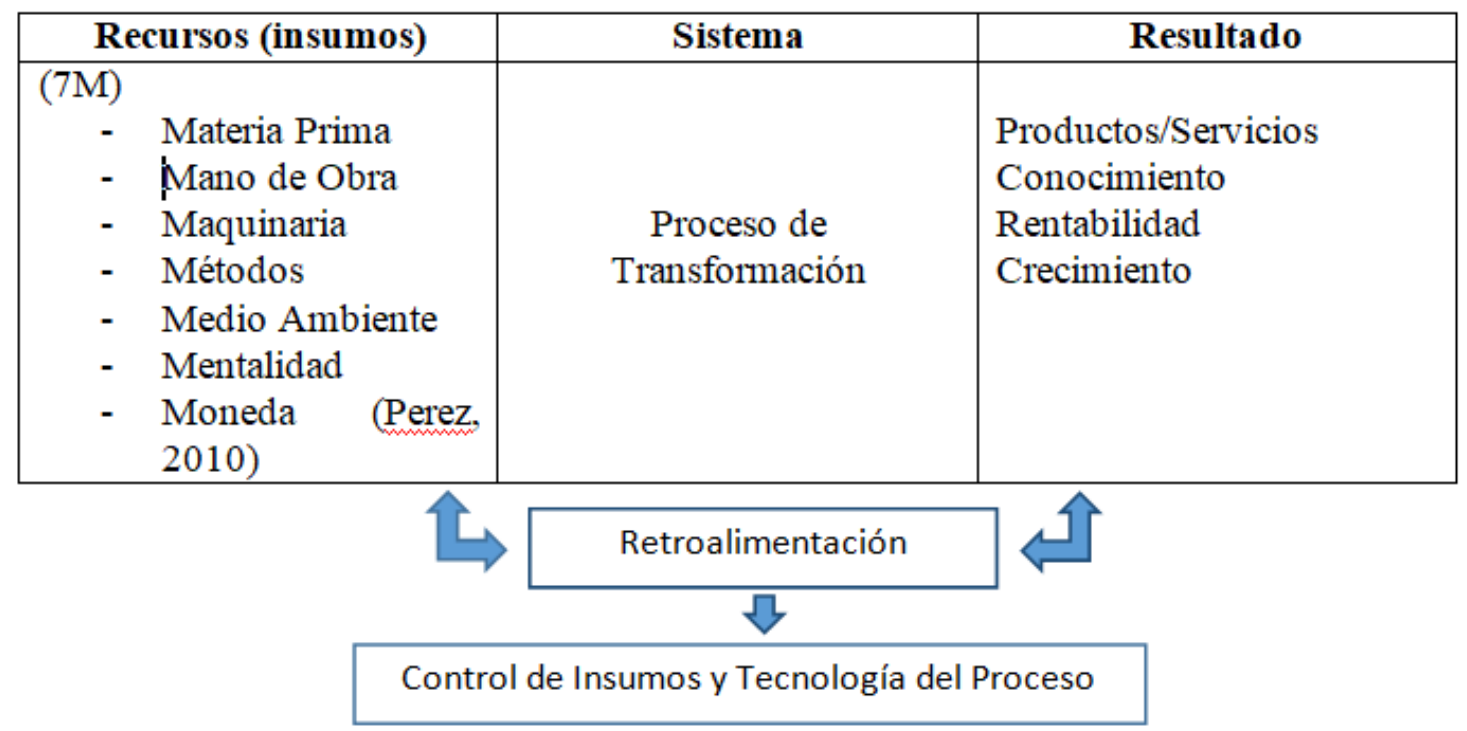

Fuente: Mantilla \& Santos (2020)

En referencia a las funciones del sistema de producción Mantilla \& Santos (2020), mencionan que el objetivo primordial en la mayoría de las organizaciones es lograr créditos para la inversión, lo que es igual a decir la obtención de beneficios económicos, los cuales pueden ser alcanzados al restar los costos al total de ingresos por ventas. 
De igual forma, comentan que el ingeniero industrial, generalmente encargado de planta, tiene como responsabilidad aumentar esos beneficios económicos a través de la reducción de los costos. Para ello es necesario que este personal cuente con las habilidades y conocimientos necesarios para llevar a cabo correctamente y subyugar las funciones, con el propósito de disminuir los costos y controlar el sistema.

En este sentido el objetivo de esta investigación se basa en analizar la planificación en la producción y su incidencia en la optimización de los procesos. Se utilizo una investigación bibliográfica para lograr los objetivos de esta investigación

\section{Metodología}

Se utilizó la metodología basada en la investigación documental, a través de la investigación bibliográfica, de documentos de carácter científico, artículos, publicaciones académicas, medios electrónicos, de diversas índoles, tomando ideas comúnmente adoptadas por los especialistas, y realizar un análisis descriptivo para el desarrollo del tema referente a la planificación en la producción y su incidencia para optimizar los procesos.

Hoy diversos factores afectan a las organizaciones en cuanto al tema de la productividad, por lo cual se hace imperante que se mantengan en constante actualización, en cuanto al uso de diferentes herramientas administrativas que contribuyan a mejorar y optimizar cada día sus procesos, en todas las áreas de la organización, dado a que el trabajo en conjunto y bien planificado ayudara a conseguir los resultados esperados.

\section{Resultados y Discusión}

De la misma manera, señalan que las funciones del sistema de producción son las mostradas en la figura 2.

\section{Figura 2}

Funciones del Sistema de Producción

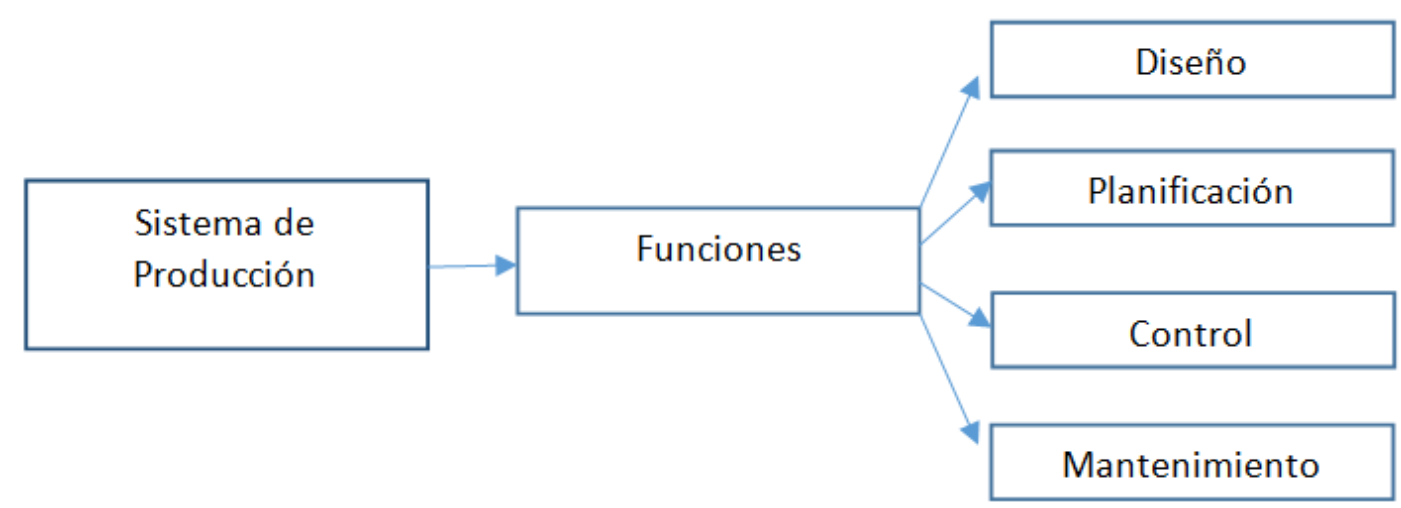

Fuente: Mantilla \& Santos (2020) 
En referencia a la función de diseño, se puede decir que, esta función incluye todas aquellas tareas como: distribución de planta, localización, recurso humano, siendo de vital importancia que este factor cuente con los conocimientos y habilidades necesarios. También es importante contar con un sistema de comunicación que permita contar con un flujo de información sobre los procesos. Asimismo, esta función, posee un lazo directo con respecto a la concepción y realización del sistema productivo. Estas funciones se pueden observar en la figura 3.

\section{Figura 3}

Función de diseño

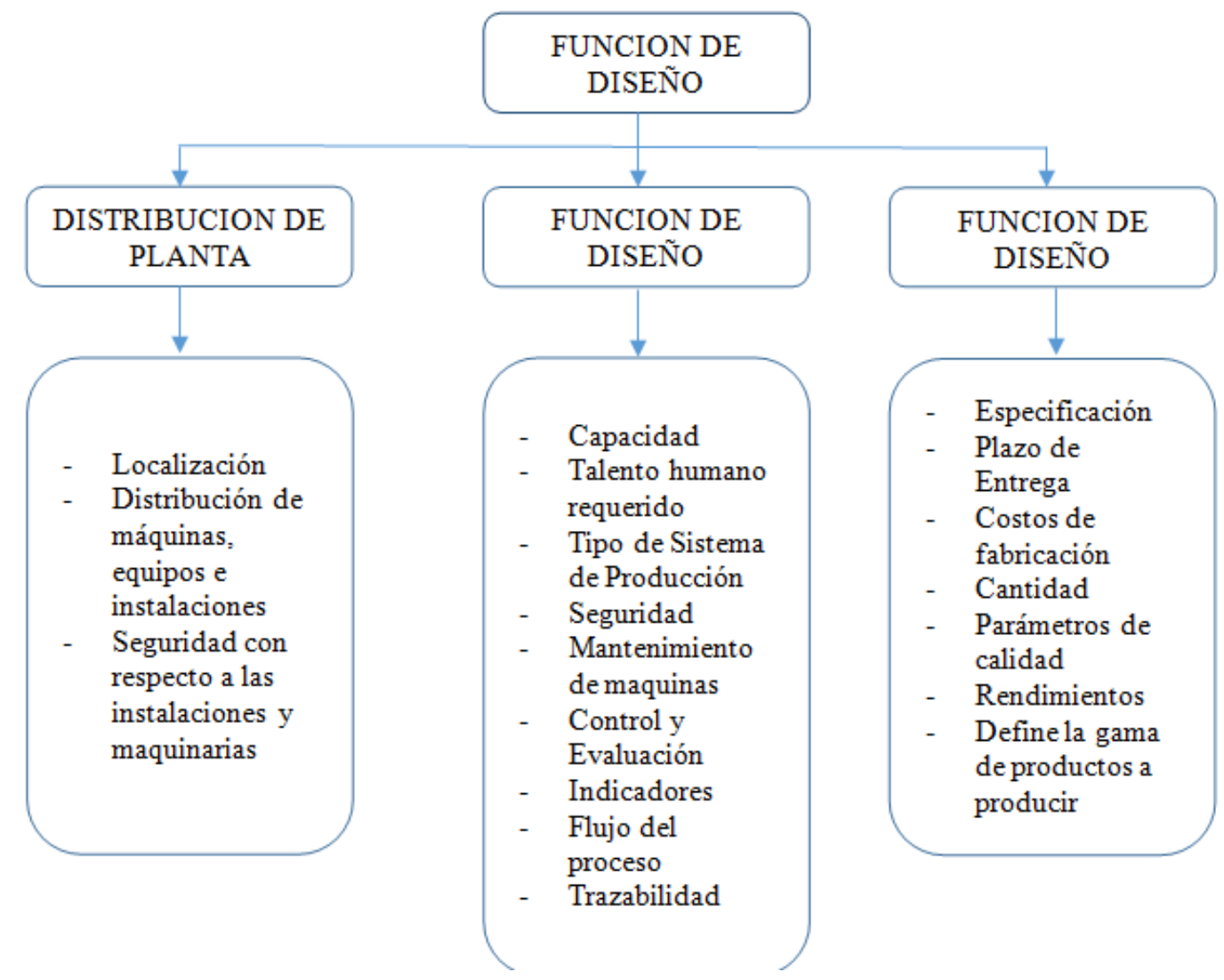

Fuente: Mantilla \& Santos (2020)

En cuanto a la función de planificación se define cuanto (volumen) y cuando (momento), se fabrica un producto, intentando mantener un equilibrio entre la producción y la capacidad del sistema que permita lograr un máximo nivel de competitividad. De allí a que sea necesario que los planes vinculen los objetivos organizacionales con los del área de producción.

Dentro de esta función, se incluyen relacionados a la gestión de operaciones como: 
- Planificación Integral: A través de esta se establecen las cantidades de productos fabricados, los niveles adecuados de personal, entre otros.

- Gestión de Inventario: Debe asegurar las existencias de los productos, con cuantos se cuenta, y cuáles deberían ser los niveles apropiados de inventario.

- Gestión de la producción: Plantea la forma de trasladar los productos y la programación de necesidades o requerimientos de materias primas.

- Gestión de cadena de suministros: Se determina como gestionar el flujo de inputs (entradas) y outputs (salidas), integrándolo a un sistema de red de proveedores, fabricantes, distribuidores y clientes.

En la figura 4, se pueden visualizar las actividades inherentes a esta función.

\section{Figura 4}

Función de la planificación

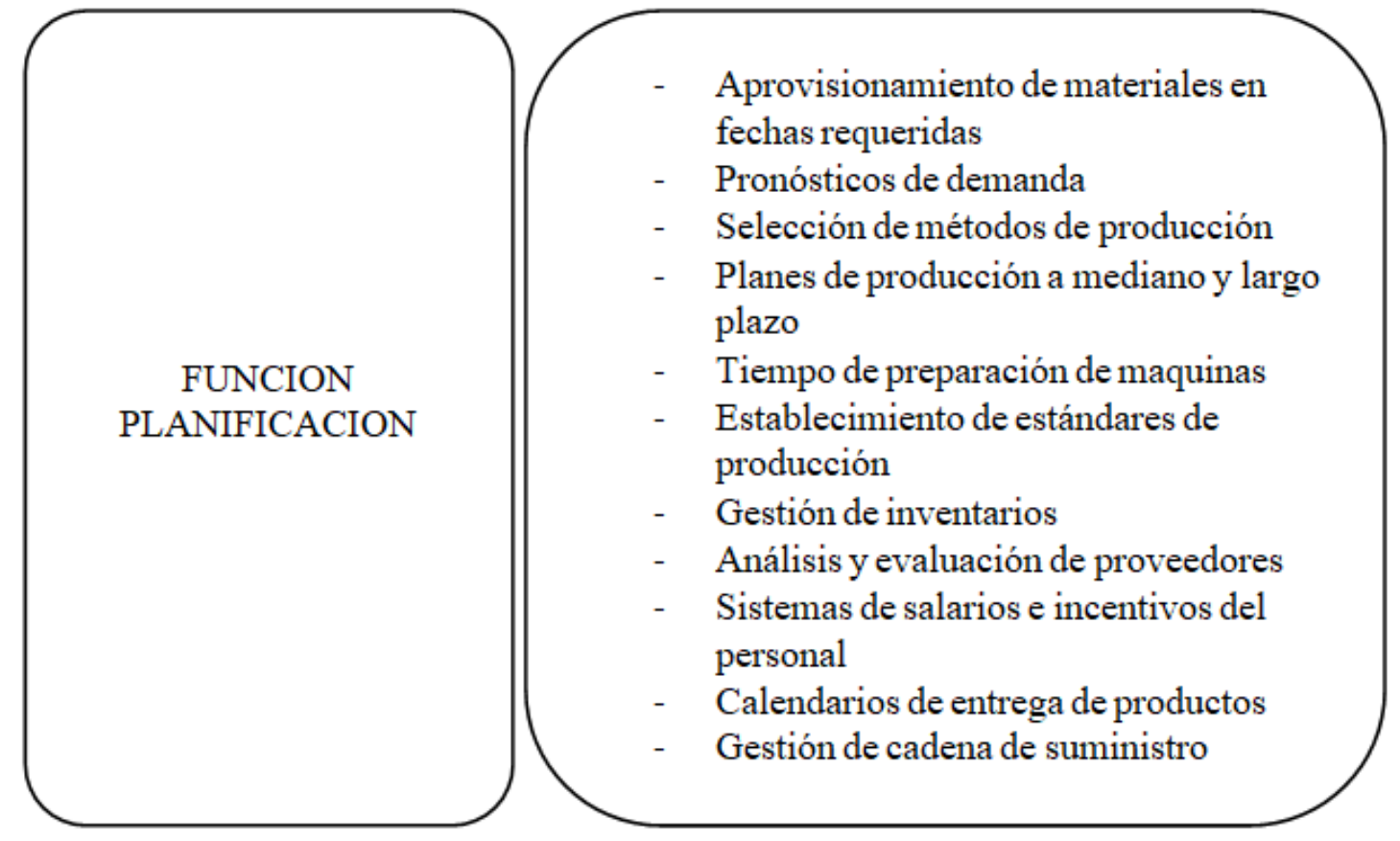

Fuente: Mantilla \& Santos (2020)

Asimismo, dentro de la función control se puede verificar si los resultados logrados están alineados con lo que se planifico, teniendo una relación ajustada con la planificación. Igualmente, esta función permite conocer aquellas desviaciones y alteraciones que se originen en los distintos procesos del sistema de producción, permitiendo así, ubicar la causa raíz del problema y aplicar las acciones correctivas necesarias a tiempo. De esta manera en la figura 5 se muestra un resume de esta función. 


\section{Figura 5}

Función de control

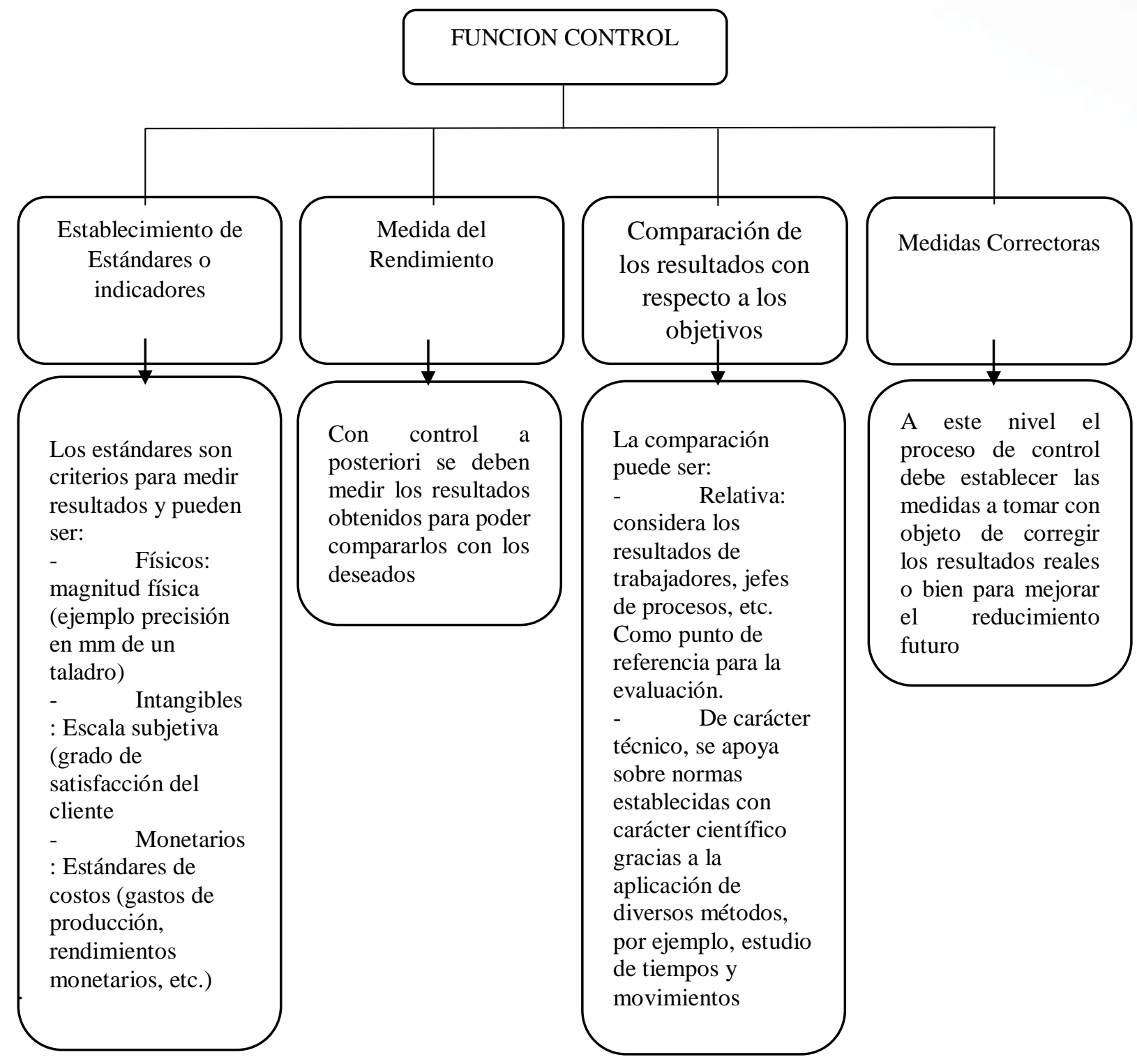

Fuente: Mantilla \& Santos (2020)

Con respecto a la función de mantenimiento, se menciona que esta permite el mantenimiento y reparación de máquinas, equipos e instalaciones, así como también, proponer la renovación de equipos y tecnologías. Por ello, esta función se involucra con todo el sistema productivo. En la figura 6 se mostrará un resume de las actividades de esta función. 


\section{Figura 6}

Función de Mantenimiento

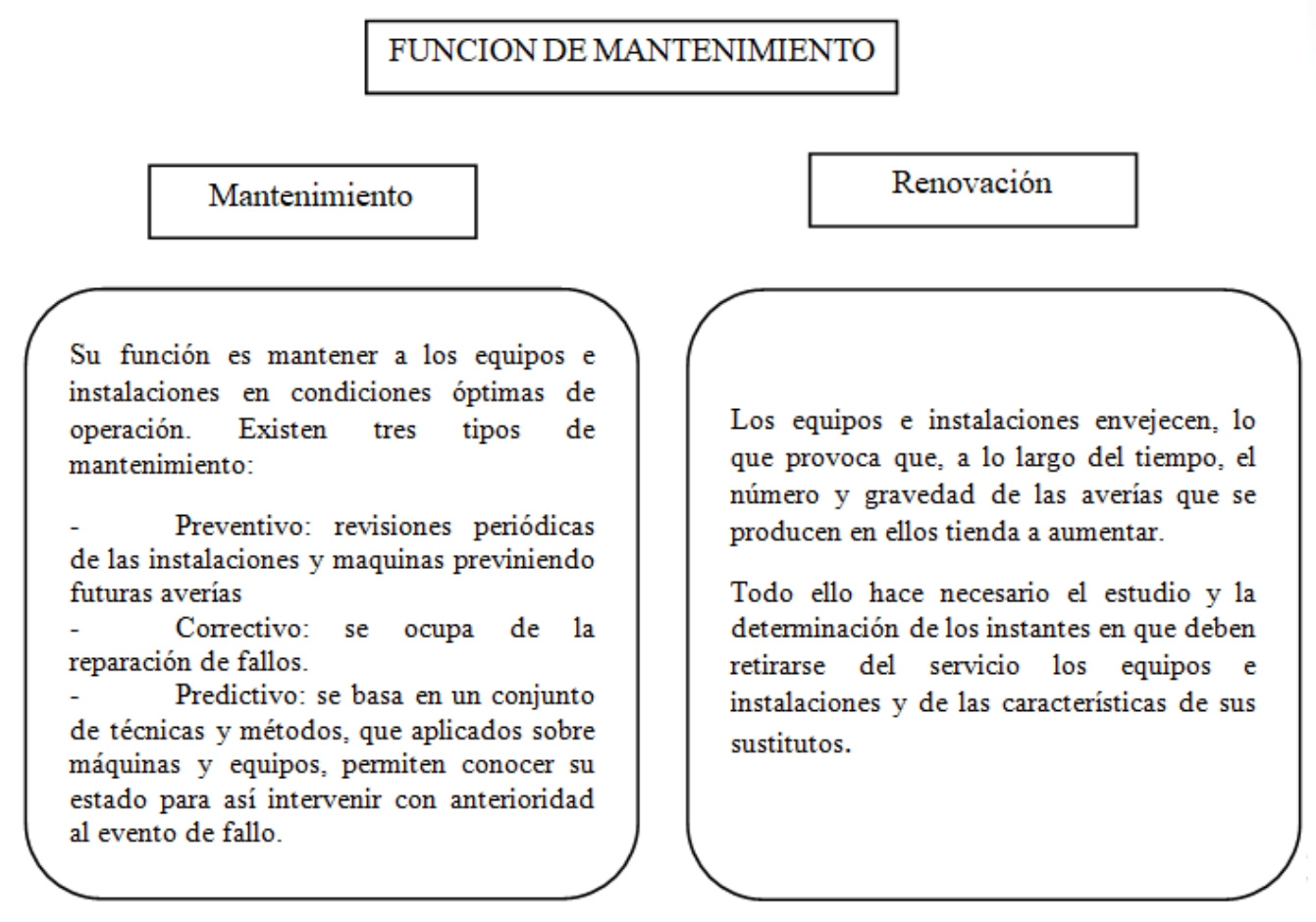

Fuente: Mantilla \& Santos (2020)

Lo anterior indica, que los sistemas de producción estas ligados estrechamente, a las distintas áreas de ingeniería presentes en una organización, ya que estas áreas se relacionan directamente con la producción, y están orientadas al manejo de las maquinarias con que cuenta la empresa, así como también, se ven directamente vinculadas al proceso de producción, en lo que se refiere a la elaboración de un producto, responsables de que cada uno de estos procesos se lleven a cabo cumpliendo los niveles de calidad, establecidos en las distintas normas internacionales como lo son las ISO, y otras tantas que rigen a las empresas de producción.

Igualmente, cada organización determinara cuál de los sistemas de producción se adecua a sus procesos internos y a la visión de la misma, de allí a la importancia de que se cumplan cada una de las funciones establecidas en el sistema de producción, ya que serán estas, las que permitan llevar a cabo diseñar una planificación y ejecución de los planes, con el propósito de obtener los resultados deseados.

De esta manera, se explica que los procesos según Iglesias (2020), son un conjunto de pasos, cuyo propósito es lograr la obtención de un producto o servicio de calidad. En este sentido Baque \& Cadena (2018), define procesos como la manera integral de identificar, comprender y aumentar el valor agregado a todos los procesos que se lleven a cabo dentro 
de la empresa, para así cumplir con las estrategias del negocio y aumentar el grado de satisfacción de los clientes.

\section{Tipos de procesos}

En referencia a los tipos de proceso Landi (2018), señala que hay tres tipos de proceso que resultan necesarios para toda organización, los cuales con:

- Procesos estratégicos: Son aquellos que están bajo la responsabilidad de los directivos de la organización, ya que son los encargados de la toma de decisiones y se encargan de buscar las estrategias que conlleven al mejoramiento de la misma, siendo su horizonte el bienestar de la compañía.

- Procesos Claves: Estos procesos son los que llevan a cabo los ejecutores, o sea, todo recurso humano que pone en práctica las estrategias diseñadas por los directivos, por lo cual deben asegurar el cumplimiento de cada una de ellas a fin de cumplir con lo requerido.

- Procesos complementarios: Son los que apoyan todos los procesos establecidos por los directivos, igualmente se da la intervención de los recursos humanos y materiales. Además, apoyaran a todos los procesos principales.

\section{Elementos de un Proceso}

En cuanto a los elementos de un proceso Achance (2018), en los procesos se presentan los siguientes elementos:

- Input (entrada): Los productos deben cumplir con las características mínimas estándar o aceptación definida. Esta entrada representa la salida de un proceso, el cual puede ser interno o externo.

- El Proceso: Representa la secuencia de actividades, así como los factores, medios $\mathrm{y}$ recursos que son necesarios para que puedan llevarse a cabo de manera eficiente. Cuenta con personal, maquinarias, equipos, tecnología, métodos de trabajo (procedimientos), información referente a calidad y entrega al cliente. De igual forma para que este proceso pueda realizarse de manera óptima y eficaz, en necesario la implementación de un sistema de control, el cual se compone de medidas e indicadores de funcionamiento del proceso, del producto y el grado de satisfacción del cliente.

- Output (salida): Es la obtención del producto con los estándares de calidad exigidos en el proceso, el cual será evaluado por el consumidor o usuario. 


\section{Planes de producción}

Según Conchillo (2021), un plan de producción es un legajo de documentos los cuales indican los pasos y tareas que deben realizarse con el propósito de transformar la materia prima en producto final. Dentro de estas tareas se pueden nombrar: aprovisionamiento, definición y orden de operaciones, tareas de limpieza, controles de calidad, medidas correctoras, entre otras.

\section{Planificación y Control de Producción}

En referencia a este apartado, Cueva (2019) señala que la planificación y control de producción no es más que un proceso a través del cual se planifica la cantidad de recursos necesarios para cubrir la demanda. La dirección que debe tomar este proceso, es el jerárquico, donde se deben integrar verticalmente los objetivos estratégicos, tácticos y operativos los cuales deben tener relación con cada una de las áreas funcionales de la organización.

Asimismo, para Collao (2004), citado por Campomanes \& Díaz (2021), el planeamiento y control de la producción, no es más que un plan de trabajo, que se acoge a los lineamientos de producción, a fin de proporcionar un alto grado de eficiencia y disminución de los costos.

Igualmente detalla, que el planeamiento y control de la producción contempla las siguientes funciones:

- Definición de las características del producto y su proceso de fabricación

- Cálculo de los costos de fabricación

- Predicción de las ventas

- Planificación y programación de la producción

- Aplicación de controles y seguimientos a la producción

Tipos de planeación de la producción:

Para López (2021), la planificación y control de la producción indica cinco fases que son:

- Planificación estrategia a largo plazo

- Planificación agregada a mediano plazo

- Programación maestra

- Programación de componentes

- Ejecución y control

La planificación representa para cualquier organización, un tema de gran relevancia, puesto que, a través de ella se pueden diseñar los planes de manera que se puedan plantear 
los objetivos que se quieren lograr, estableciendo estrategias, recursos a utilizar (personal, materiales, insumos, tecnología, etc.)

Esta herramienta, es utilizada en las distintas áreas de una organización, aplicable tanto a las áreas administrativas como en las áreas de producción, ya que, a través de esta, se pueden diseñar desde las estrategias hasta los recursos con que se llevara a cabo, alineando dichas estrategias con los lineamientos organizacionales.

La combinación de la planificación de la producción, no es más que el diseño de planes que se relacionan directamente con esta área, y que busca optimizar cada uno de los procesos productivos, para poder obtener los resultados esperados.

La implementación de un buen plan de producción, permitirá la realización de cada una de las tareas, de una manera eficiente y eficaz, con el propósito de poder garantizar un producto o servicio de alta calidad, y que cumpla con las necesidades y expectativas de los consumidores.

\section{Conclusiones}

Es primordial que las organizaciones conozcan la capacidad de producción que tienen, ya que esto permitirá una eficaz planificación de la producción y ventas, lo cual permitirá conocer la oferta de los productos y servicios que se comercializaran.

De igual forma, resulta relevante implementar una gestión eficaz de planificación que se ajuste al comportamiento de los mercados y al alto grado de competitividad que existe.

El volumen de producción de una organización representa un factor preciso, para dirigir de manera efectiva el portafolio de productos, según sus estándares de estructura, calidad, cantidad y oportunidad, tomando en cuenta las exigencias de los consumidores.

Por otra parte, la planeación permitirá ejecutar las medidas necesarias con el propósito de comparar los sistemas de producción con los requerimientos futuros, usando técnicas de proyección de la demanda, lo cual requiere de una planeación a corto, mediano y largo plazo y con ello satisfacer lo requerido por los consumidores.

Entonces, la aplicación y ejecución de un buen plan de producción, permitirá la venta de productos o prestación de servicios que arrojara resultados positivos que podrán observarse al momento de obtener las ganancias, de allí a que resulte importante que las organizaciones puedan ser capaz de saber qué cantidad es capaz de producir, y de esta forma conocer cuánto dispone para vender.

Para ello entonces en necesario que las organizaciones puedan dar respuesta a preguntas como: 
- Cuál es la capacidad de operación de la empresa?

- ¿Qué cantidad de unidades de un producto o servicio son necesarias para generar ingresos?

- ¿Si es necesario renovar la planta tecnológica?

- ¿Si se cuenta con mano de obra especializada y capacitada o por el contrario se hace necesario la contratación de nuevos talentos?

- ¿Innovar en la producción de nuevos productos?

- ¿Si es necesario la renovación de maquinarias y equipos?

- ¿Identificar la actividad más importante dentro del proceso?

Las repuestas a estas interrogantes, pueden permitir el diseño de una buena planificación de producción y por ende la fijación de estrategias que contribuyan a la obtención de resultados positivos, que le permitan a la organización alcanzar sus metas financieras y posicionarse dentro del mercado.

\section{Referencias Bibliográficas}

Achance, W. (2018). Estandarización de procesos en la línea de producción de Balanceado de pollos en la empresa Molinos Anita, para incrementar la productividad. Riobamba, Ecuador: Trabajo especial de grado de la Universidad Nacional de Chimborazo para optar al título de Ingeniero Industrial.

Alvarado, F. (2018). Planificación de los procesos de producción del vino en la vitivinícola San Leonardo Chincha-ICA, 2018. Perú: Trabajo especial de grado de la Universidad César Vallejo.

Baque, P., \& Cadena, D. (2018). Elaboración de una propuesta de mejora de los procesos en el área de producción de la empresa Mafrico S.A de la Ciudad de Guayaquil. Guayaquil, Ecuador: Trabajo especial de grado de la Universidad de Guayaquil.

Campomanes, Y., \& Díaz, C. (2021). Propuesta de un proceso de planeamiento, control y ejecución de la producción basado en el modelo $S \& O P$, mediante herramientas de gestión de procesos para mejorar la productividad de las empresas pymes del sector productor de chirimoyas. Perú: Trabajo especial de grado de la Universidad Peruana de Ciencias Aplicadas.

Conchillo, L. (2021). Planificación de la producción del Valaq Patrol. España: Trabajo especial de grado de la Universidad Politécnica de València.

Cueva, J. (2019). Implementación de un modelo de planificación de la producción y presupuestos agrícolas en Agro Casagrande SAC. Perú: Trabajo especial de grado de la Universidad Nacional de Trujillo. 
Encalada-Tenorio, G., Sandoya-Valero, E., Garofalo-Velasco, D., \& Troya-Terranova, T. (2021). Importancia de la planificación como herramienta para anticipar decisiones en una empresa de servicios. FIPCAEP Enfoques 6(1), 190-201.

Iglesias, M. (2020). Implementación del control interno en el área de producción y su incidencia en la gestión de los inventarios de la empresa de servicio grafico Urbano SRL, Chorrillos 2018. Perú: Trabajo especial de grado de la Universidad Peruana las Américas.

Landi, X. (2018). Propuesta de mejora de los procesos de producción de la empresa VID, en la ciudad de Guayaquil. Ecuador: Trabajo especial de grado de la Universidad de Guayaquil.

López, E. (2021). Programa de producción para la optimización de recursos materiales en la empresa Ralomtex. Ecuador: Trabajo especial de grado de la Pontificia Universidad Católica del Ecuador Sede Ambato.

Mantilla, G., \& Santos, L. (2020). Propuesta de mejora en el área de producción y manteamiento para reducir los costos operativos en una empresa metalmecánica de la ciudad de Trujillo. Perú: Trabajo especial de grado de la Universidad Privada del Norte.

Muñoz, J. (2019). Planificación de la producción en la empresa Olimphico Sports del Cantón Cayambe. Trabajo especial de grado de la Universidad Técnica del Norte.

Pinzón, B. (2018). Optimización de la planificación para la producción de tejido de punto en la empresa $R \&$ L Textiles SAC, Lima 2018. Trabajo especial de grado de la Universidad Norbert Wiener.

Solís-Ferrer, H., \& Cortez-Fajardo, M. (2020). La importancia de la planificación de la producción en una empresa de conformado con PVC. Polo del Conocimiento 5(10), 440-457.

Villamarin, G. (2021). Evaluación del impacto financiero por la falta de implementación de un sistema de costos por órdenes de producción en la empresa "Creaciones Edwin S”, JR, durante el periodo del año 2020-2021. Ecuador: Trabajo especial de grado de la Universidad Politécnica Salesiana.

\section{Ciencia Ligital}


El artículo que se publica es de exclusiva responsabilidad de los autores y no necesariamente reflejan el pensamiento de la Revista Alfa Publicaciones.

\section{Ciencia}

El artículo queda en propiedad de la revista y, por tanto, su publicación parcial y/o total en otro medio tiene que ser autorizado por el director de la Revista Alfa Publicaciones.
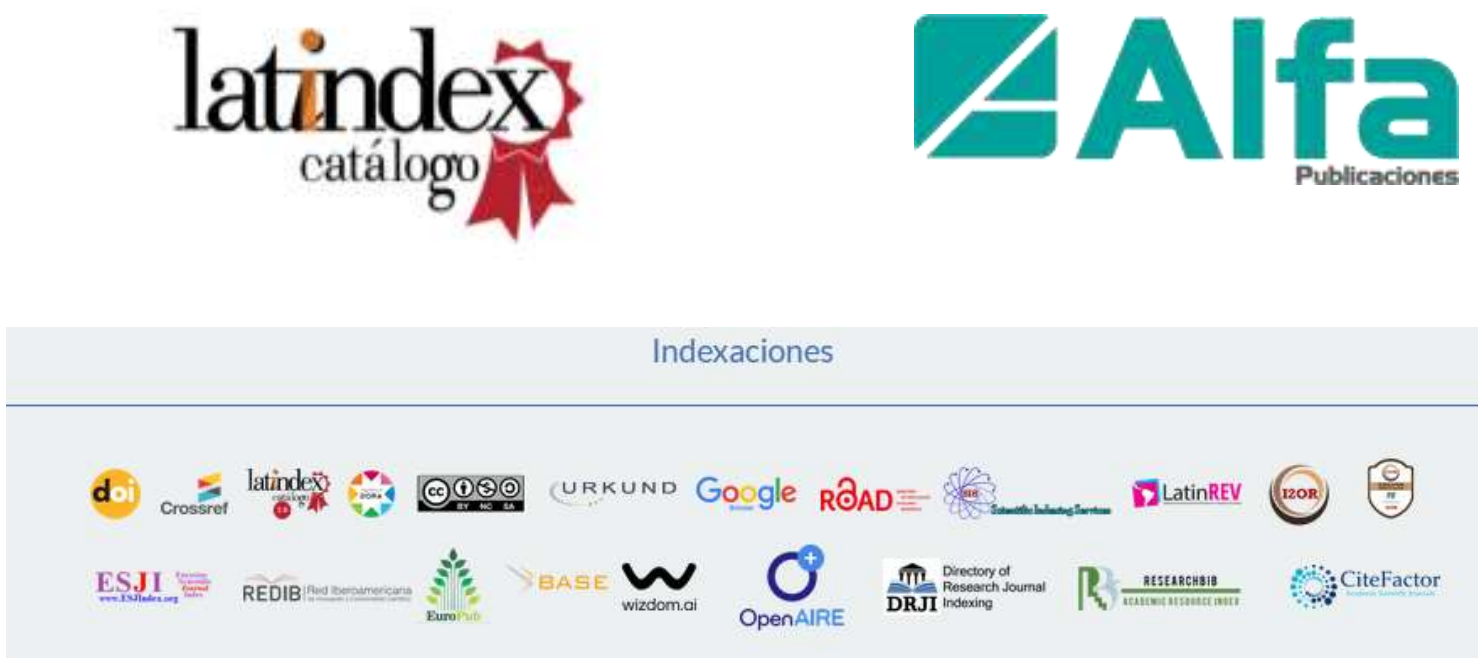